\title{
Author Index Volume 30 (2012)
}

The issue number is given in front of the pagination

Aalten, P., see Gommer, E.D. (4) 805-813

Aimar, P., see Alasia, S. (1) 41-51

Akrivos, J., see Ravona-Springer, R. (2) 299-309

Alasia, S., P. Aimar, A. Merighi and L. Lossi, ContextDependent Toxicity of Amyloid- $\beta$ Peptides on Mouse Cerebellar Cells (1) 41-51

Albani, D., F.M. Boneschi, G. Biella, G. Giacalone, S. Lupoli, F. Clerici, L. Benussi, R. Ghidoni, D. Galimberti, R. Squitti, S. Mariani, A. Confaloni, G. Bruno, C. Mariani, E. Scarpini, G. Binetti, G. Magnani, M. Franceschi and G. Forloni, Replication Study to Confirm the Role of CYP2D6 Polymorphism rs1080985 on Donepezil Efficacy in Alzheimer's Disease Patients (4) 745-749

Aldudo, J., see Santana, S. (4) 815-831

Alegret, M., G. Cuberas-Borrós, G. Vinyes-Junqué, A. Espinosa, S. Valero, I. Hernández, I. Roca, A. Ruíz, M. Rosende-Roca, A. Mauleón, J.T. Becker, J. Castell-Conesa, L. Tárraga and M. Boada, A Two-Year Follow-Up of Cognitive Deficits and Brain Perfusion in Mild Cognitive Impairment and Mild Alzheimer's Disease (1) 109-120

Alquézar, C., see Esteras, N. (2) 337-353

Amit, T., see Zheng, H. (1) 1-16

Ancelin, M.-L., I. Carrière, P. Barberger-Gateau, S. Auriacombe, O. Rouaud, S. Fourlanos, C. Berr, A.-M. Dupuy and K. Ritchie, Lipid Lowering Agents, Cognitive Decline, and Dementia: The Three-City Study (3) 629-637

Anderson, K.L., see Searcy, J.L. (4) 943-961

Andreasson, U., see Mattsson, N. (4) 767-778

Anstey, K.J., see Bunce, D. (4) 935-942

Antequera, D., see Esteras, N. (2) 337-353

Antonell, A., see Balasa, M. (3) 605-616

Arendash, G.W., see Cao, C. (3) 559-572

Armato, U., see Chakravarthy, B. (3) 675-684

Arroyo-Anlló, E.M.,P. Ingrand and R. Gil,Improvement of Semantic Categorization through Procedural Learning in Alzheimer's Disease (1) 121-129

Arseneault, M., see Doggui, S. (2) 377-392

Ashok, R., see Cao, C. (3) 559-572
Aso, E., E. Palomer, S. Juvés, R. Maldonado, F.J. Muñoz and I. Ferrer, CB 1 Agonist ACEA Protects Neurons and Reduces the Cognitive Impairment of A $\beta P P / P S 1$ Mice (2) 439-459

Auriacombe, S., see Ancelin, M.-L. (3) 629-637

Bai, G., see Cao, C. (3) 559-572

Bai, X., see Wang, X. (3) 665-673

Balasa, M., D. Vidal-Piñeiro, A. Lladó, A. Antonell, B. Bosch, F. Castellanos, N. Bargalló, D. Bartres-Faz, J.-L. Molinuevo and R. SánchezValle, PSEN1 Mutation Carriers Present Lower Cerebrospinal Fluid Amyoid- $\beta_{42}$ Levels than Sporadic Early-Onset Alzheimer's Disease Patients but no Differences in Neuronal Injury Biomarkers (3) 605-616

Balastik, M., see Pastorino, L. (2) 277-297

Bangen, K.J., see Nation, D.A. (3) 595-603

Bar-Am, O., see Zheng, H. (1) 1-16

Barberger-Gateau, P., see Ancelin, M.-L. (3) 629-637

Bargalló, N., see Balasa, M. (3) 605-616

Barnham, K.J., see Watt, A.D. (2) 323-336

Barrios, L., see Esteras, N. (2) 337-353

Bartolomé, F., see Esteras, N. (2) 337-353

Bartres-Faz, D., see Balasa, M. (3) 605-616

Baudry, M., see Clausen, A. (1) 183-208

Becker, J.T., see Alegret, M. (1) 109-120

Becker, J.T., see Hughes, T.M. (1) 53-61

Beckett, T.L., see Searcy, J.L. (4) 943-961

Beeri, M.S., see Ravona-Springer, R. (2) 299-309

Benussi, L., see Albani, D. (4) 745-749

Berr, C., see Ancelin, M.-L. (3) 629-637

Besengez, C., see Do, T.M. (1) 155-166

Bhoiwala, D.L., see Mathew, A. (3) 617-627

Bi, J., see Wei, L. (3) 531-543

Bi, X., see Clausen, A. (1) 183-208

Biella, G., see Albani, D. (4) 745-749

Binetti, G., see Albani, D. (4) 745-749

Biundo, F., see Buoso, E. (2) 393-405

Blalock, E.M., see Searcy, J.L. (4) 943-961

Blennow, K., see Mattsson, N. (4) 767-778

Boada, M., see Alegret, M. (1) 109-120 
Bombois, S., see Rollin-Sillaire, A. (4) 833-845

Bondi, M.W., see Nation, D.A. (3) 595-603

Boneschi, F.M., see Albani, D. (4) 745-749

Borbon, I., J. Totenhagen, M.T. Fiorenza, S. Canterini, W. Ke, T. Trouard and R.P. Erickson, NiemannPick C1 Mice, a Model of “Juvenile Alzheimer's Disease", with Normal Gene Expression in Neurons and Fibrillary Astrocytes Show Long Term Survival and Delayed Neurodegeneration (4) $875-887$

Borenstein, A.R., see Mortimer, J.A. (4) 757-766

Borowczyk, K., D.M. Shih and H. Jakubowski, Metabolism and Neurotoxicity of Homocysteine Thiolactone in Mice: Evidence for a Protective Role of Paraoxonase 1 (2) 225-231

Bosch, B., see Balasa, M. (3) 605-616

Boucau, M.-C., see Saint-Pol, J. (3) 489-503

Bourasset, F., see Do, T.M. (1) 155-166

Boutoleau-Bretonnière, C., see Wallon, D. (4) 847-856

Brandel, J.-P., see Schmidt, C. (4) 751-756

Brickman, A.M., see Geerlings, M.I. (1) 75-82

Brouillette, R.M., see Bruce-Keller, A.J. (4) 899-908

Brown, A.J., see Sharpe, L.J. (1) 35-39

Bruce-Keller, A.J., R.M. Brouillette, C. Tudor-Locke, H.C. Foil, W.P. Gahan, D.M. Nye, L. Guillory and J.N. Keller, Relationship Between Cognitive Domains, Physical Performance, and Gait in Elderly and Demented Subjects (4) 899-908

Bruno, G., see Albani, D. (4) 745-749

Bucossi, S., see Ventriglia, M. (4) 981-984

Bullido, M.J., see Santana, S. (4) 815-831

Bunce, D., K.J. Anstey, N. Cherbuin, P. Gautam, P. Sachdev and S. Easteal, APOE Genotype and Entorhinal Cortex Volume in Non-Demented Community-Dwelling Adults in Midlife and Early Old Age (4) 935-942

Buoso, E., F. Biundo, C. Lanni, G. Schettini, S. Govoni and M. Racchi, A $\beta$ PP Intracellular C-Terminal Domain Function is Related to its Degradation Processes (2) 393-405

Burgmans, S., see Echávarri, C. (4) 909-917

Burgos, J.S., see Guzman-Sanchez, F. (4) 779-790

Buyse, M., see Do, T.M. (1) 155-166

Caballero, M.C., see Echávarri, C. (4) 909-917

Cai, J., see Cao, C. (3) 559-572

Calero-Lara, M., see Schmidt, C. (4) 751-756

Calon, F., see Do, T.M. (1) 155-166

Camins, A., see Casadesús, G. (3) 573-583

Campion, D., see Wallon, D. (4) 847-856

Candela, P., see Saint-Pol, J. (3) 489-503
Canterini, S., see Borbon, I. (4) 875-887

Cao, C., D.A. Loewenstein, X. Lin, C. Zhang, L. Wang, R. Duara, Y. Wu, A. Giannini, G. Bai, J. Cai, M. Greig, E. Schofield, R. Ashok, B. Small, H. Potter and G.W. Arendash, High Blood Caffeine Levels in MCI Linked to Lack of Progression to Dementia (3) $559-572$

Carrière, I., see Ancelin, M.-L. (3) 629-637

Carro, E., see Esteras, N. (2) 337-353

Casadesús, G., J. Gutierrez-Cuesta, H.-g. Lee, A. Jiménez, M. Tajes, D. Ortuño-Sahagún, A. Camins, M.A. Smith and M. Pallàs, Neuronal Cell Cycle Re-Entry Markers are Altered in the Senescence Accelerated Mouse P8 (SAMP8) (3) 573-583

Castellanos, F., see Balasa, M. (3) 605-616

Castell-Conesa, J., see Alegret, M. (1) 109-120

Cavaco, S., see Taipa, R. (1) 83-90

Cecchelli, R., see Saint-Pol, J. (3) 489-503

Cerdán, S., see Esteras, N. (2) 337-353

Chacun, H., see Do, T.M. (1) 155-166

Chakravarthy, B., M. Ménard, S. Ito, C. Gaudet, I.D. Prá, U. Armato and J. Whitfield, Hippocampal Membrane-Associated p $75^{\mathrm{NTR}}$ Levels are Increased in Alzheimer's Disease (3) 675-684

Chapa, R.D.P., see Fawver, J.N. (1) 63-73

Chávez, R., see Diaz, A. (3) 505-522

Checler, F., see Flammang, B. (1) 145-153

Chen, K.-C., see Searcy, J.L. (4) 943-961

Chen, N.-N., D.-J. Luo, X.-Q. Yao, C. Yu, Y. Wang, Q. Wang, J.-Z. Wang and G.-P. Liu, Pesticides Induce Spatial Memory Deficits with Synaptic Impairments and an Imbalanced Tau Phosphorylation in Rats (3) 585-594

Chen, W.-T., see Wang, P.-N. (2) 423-437

Cherbuin, N., see Bunce, D. (4) 935-942

Chimini, G., see Do, T.M. (1) 155-166

Chiu, A.W.L., see Zhang, C. (4) 919-934

Chou, K.-H., see Wang, P.-N. (2) 423-437

Chu, S., see Mortimer, J.A. (4) 757-766

Chui, D., see Yu, Y. (2) 263-275

Cisternino, S., see Do, T.M. (1) 155-166

Clarkson, M.J., see Rohrer, J.D. (2) 407-411

Clausen, A., X. Xu, X. Bi and M. Baudry, Effects of the Superoxide Dismutase/Catalase Mimetic EUK207 in a Mouse Model of Alzheimer's Disease: Protection Against and Interruption of Progression of Amyloid and Tau Pathology and Cognitive Decline (1) 183-208

Clerici, F., see Albani, D. (4) 745-749

Confaloni, A., see Albani, D. (4) 745-749

Coppola, G., see Lunnon, K. (3) 685-710 
Crawford, D.R., see Mathew, A. (3) 617-627

Crofton, A., see Zabel, M. (2) 311-321

Croisile, B., see Wallon, D. (4) 847-856

Cuberas-Borrós, G., see Alegret, M. (1) 109-120

Dabert-Gay, A.-S., see Flammang, B. (1) 145-153

Damásio, J., see Taipa, R. (1) 83-90

Dao, L., see Doggui, S. (2) 377-392

Dasari, B., see Prasanthi, J.R.P. (1) 167-182

de Assis, A.M., see Muller, A.P. (4) 889-898

de Pedro-Cuesta, J., see Schmidt, C. (4) 751-756

Deary, I.J., see Whalley, L.J. (2) 253-261

Debayle, D., see Flammang, B. (1) 145-153

DeCarli, C., see Mortimer, J.A. (4) 757-766

Dehouck, L., see Saint-Pol, J. (3) 489-503

Dehouck, M.-P., see Saint-Pol, J. (3) 489-503

del Valle, E., see Martínez, E. (2) 233-244

Delano-Wood, L., see Nation, D.A. (3) 595-603

Delisle, M.-B., see Wallon, D. (4) 847-856

Deramecourt, V., see Rollin-Sillaire, A. (4) 833-845

Devanand, D.P., see Geerlings, M.I. (1) 75-82

Diamond, K., see Mowszowski, L. (1) 209-219

Diaz, A., D. Limon, R. Chávez, E. Zenteno and J. Guevara, $A \beta_{25-35}$ Injection into the Temporal Cortex Induces Chronic Inflammation that Contributes to Neurodegeneration and Spatial Memory Impairment in Rats (3) 505-522

Dickson, A., see Prasanthi, J.R.P. (1) 167-182

Dickson, A., see Zabel, M. (2) 311-321

Ding, D., see Mortimer, J.A. (4) 757-766

Do, T.M., M.-S. Noel-Hudson, S. Ribes, C. Besengez, M. Smirnova, S. Cisternino, M. Buyse, F. Calon, G. Chimini, H. Chacun, J.-M. Scherrmann, R. Farinotti and F. Bourasset, ABCG2- and ABCG4Mediated Efflux of Amyloid- $\beta$ Peptide (1-40) at the Mouse Blood-Brain Barrier (1) 155-166

Dobson, R., see Lunnon, K. (3) 685-710

Doggui, S., J.K. Sahni, M. Arseneault, L. Dao and C. Ramassamy, Neuronal Uptake and Neuroprotective Effect of Curcumin-Loaded PLGA Nanoparticles on the Human SK-N-SH Cell Line (2) 377-392

Donath, A., see Herring, A. (4) 963-979

Duara, R., see Cao, C. (3) 559-572

Duchesne, S., see Mouiha, A. (1) 91-100

Dupuy, A.-M., see Ancelin, M.-L. (3) 629-637

Duthie, A.C., see Whalley, L.J. (2) 253-261

Duyckaerts, C., see Wallon, D. (4) 847-856

Easteal, S., see Bunce, D. (4) 935-942

Echávarri, C., S. Burgmans, M.C. Caballero, F. GarcíaBragado, F.R.J. Verhey and H.B.M. Uylings, Co- occurrence of Different Pathologies in Dementia: Implications for Dementia Diagnosis (4) 909-917

ElAli, A., see Herring, A. (4) 963-979

Erickson, R.P., see Borbon, I. (4) 875-887

Espinosa, A., see Alegret, M. (1) 109-120

Esteras, N., C. Alquézar, F. Bartolomé, D. Antequera, L. Barrios, E. Carro, S. Cerdán and Á. MartínRequero, Systematic Evaluation of Magnetic Resonance Imaging and Spectroscopy Techniques for Imaging a Transgenic Model of Alzheimer's Disease (A $\beta$ PP/PS1) (2) 337-353

Evans, R.W., see Hughes, T.M. (1) 53-61

Fan, D.S., see Yu, Y. (2) 263-275

Farinotti, R., see Do, T.M. (1) 155-166

Fawver, J.N., H.E. Schall, R.D.P. Chapa, X. Zhu and I.V.J. Murray, Amyloid- $\beta$ Metabolite Sensing: Biochemical Linking of Glycation Modification and Misfolding (1) 63-73

Fenart, L., see Saint-Pol, J. (3) 489-503

Feng, J., see Zhang, C. (4) 919-934

Ferrer, I., see Aso, E. (2) 439-459

Fiorenza, M.T., see Borbon, I. (4) 875-887

Flammang, B., R. Pardossi-Piquard, J. Sevalle, D. Debayle, A.-S. Dabert-Gay, A. Thévenet, I. Lauritzen and F. Checler, Evidence that the Amyloid- $\beta$ Protein Precursor Intracellular Domain, AICD, Derives From $\beta$-Secretase-Generated C-Terminal Fragment (1) 145-153

Foil, H.C., see Bruce-Keller, A.J. (4) 899-908

Forloni, G., see Albani, D. (4) 745-749

Fourlanos, S., see Ancelin, M.-L. (3) 629-637

Fox, H.C., see Whalley, L.J. (2) 253-261

Fox, N.C., see Rohrer, J.D. (2) 407-411

Franceschi, M., see Albani, D. (4) 745-749

Frebourg, T., see Wallon, D. (4) 847-856

Fridkin, M., see Zheng, H. (1) 1-16

Friedland, R.P., S. Nandi, Book Review (3) 729-730

Friedland, R.P., see Sabbagh, M.N. (3) 711-727

Fukushim, M., see Zhou, B. (2) 367-375

Furney, S., see Lunnon, K. (3) 685-710

Gabelle, A., see Wallon, D. (4) 847-856

Gahan, W.P., see Bruce-Keller, A.J. (4) 899-908

Galasko, D.R., see Nation, D.A. (3) 595-603

Galimberti, D., see Albani, D. (4) 745-749

Galimberti, D., see Taipa, R. (1) 83-90

Galina, A., see Muller, A.P. (4) 889-898

García-Bragado, F., see Echávarri, C. (4) 909-917

Garner, B., see Sharpe, L.J. (1) 35-39

Gaudet, C., see Chakravarthy, B. (3) 675-684 
Gautam, P., see Bunce, D. (4) 935-942

Ge, J., see Yu, Y. (2) 263-275

Geerlings, M.I., A.M. Brickman, N. Schupf, D.P. Devanand, J.A. Luchsinger, R. Mayeux and S.A. Small, Depressive Symptoms, Antidepressant Use, and Brain Volumes on MRI in a PopulationBased Cohort of Old Persons Without Dementia (1) $75-82$

Geschwind, D., see Lunnon, K. (3) 685-710

Geula, C., see Sabbagh, M.N. (3) 711-727

Ghidoni, R., see Albani, D. (4) 745-749

Ghribi, O., see Prasanthi, J.R.P. (1) 167-182

Giacalone, G., see Albani, D. (4) 745-749

Giannini, A., see Cao, C. (3) 559-572

Gil, R., see Arroyo-Anlló, E.M. (1) 121-129

Godbold, J., see Ravona-Springer, R. (2) 299-309

Gommer, E.D., E.G.H.J. Martens, P. Aalten, E. Shijaku, F.R.J. Verhey, W.H. Mess, I.H.G.B. Ramakers and J.P.H. Reulen, Dynamic Cerebral Autoregulation in Subjects with Alzheimer's Disease, Mild Cognitive Impairment, and Controls: Evidence for Increased Peripheral Vascular Resistance with Possible Predictive Value (4) 805-813

Gosselet, F., see Saint-Pol, J. (3) 489-503

Govoni, S., see Buoso, E. (2) 393-405

Graham, R.M., see Johnstone, D.M. (4) 791-803

Greig, M., see Cao, C. (3) 559-572

Grossman, H.T., see Ravona-Springer, R. (2) 299-309

Guevara, J., see Diaz, A. (3) 505-522

Guillory, L., see Bruce-Keller, A.J. (4) 899-908

Guo, Q., see Mortimer, J.A. (4) 757-766

Gustavsson, M., see Mattsson, N. (4) 767-778

Gutierrez-Cuesta, J., see Casadesús, G. (3) 573-583

Guyant-Maréchal, L., see Wallon, D. (4) 847-856

Guzman-Sanchez, F., F. Valdivieso and J.S. Burgos, Aging-Related Neurostructural, Neuropathological, and Behavioral Changes Associated with Herpes Simplex Virus Type 1 Brain Infection in Mice (4) 779-790

Haas, C.B., see Muller, A.P. (4) 889-898

Haik, S., see Schmidt, C. (4) 751-756

Halliday, G.M., see Sharpe, L.J. (1) 35-39

Hallikainen, M., see Hartikainen, P. (4) 857-874

Hamzehian, S., see Herring, A. (4) 963-979

Hannequin, D., see Wallon, D. (4) 847-856

Hansen, L.A., see Nation, D.A. (3) 595-603

Haroutunian, V., see Ravona-Springer, R. (2) 299-309

Hartikainen, P., J. Räsänen, V. Julkunen, E. Niskanen, M. Hallikainen, M. Kivipelto, R. Vanninen, A.M. Remes and H. Soininen, Cortical Thickness in Frontotemporal Dementia, Mild Cognitive Impairment, and Alzheimer's Disease (4) 857874

Hauw, J.-J., see Schmidt, C. (4) 751-756

He, P., see Long, J. (3) 545-558

Hermann, D.M., see Herring, A. (4) 963-979

Hermens, D.F., see Mowszowski, L. (1) 209-219

Hernández, I., see Alegret, M. (1) 109-120

Herring, A., A. Donath, K.M. Steiner, M.P. Widera, S. Hamzehian, D. Kanakis, K. Kölble, A. ElAli, D.M. Hermann, W. Paulus and K. Keyvani, Reelin Depletion is an Early Phenomenon of Alzheimer's Pathology (4) 963-979

Hickie, I.B., see Mowszowski, L. (1) 209-219

Hodges, A., see Lunnon, K. (3) 685-710

Huang, P., see Pastorino, L. (2) 277-297

Hughes, T.M., L.H. Kuller, O.L. Lopez, J.T. Becker, R.W. Evans, K. Sutton-Tyrrell and C. Rosano, Markers of Cholesterol Metabolism in the Brain Show Stronger Associations with Cerebrovascular Disease than Alzheimer's Disease (1) 53-61

Hui, C., see Yu, Y. (2) 263-275

Hushmendy, S.F., see Mathew, A. (3) 617-627

Ibrahim, Z., see Lunnon, K. (3) 685-710

Ingrand, P., see Arroyo-Anlló, E.M. (1) 121-129

Isaac, M., see Klugman, A. (3) 467-474

Ito, S., see Chakravarthy, B. (3) 675-684

Jak, A.J., see Nation, D.A. (3) 595-603

Jakubowski, H., see Borowczyk, K. (2) 225-231

Jiménez, A., see Casadesús, G. (3) 573-583

Johnstone, D.M., R.M. Graham, D. Trinder, C. Riveros, J.K. Olynyk, R.J. Scott, P. Moscato and E.A. Milward, Changes in Brain Transcripts Related to Alzheimer's Disease in a Model of HFE Hemochromatosis are not Consistent with Increased Alzheimer's Disease Risk (4) 791-803

Jouanny, P., see Serot, J.-M. (1) 17-26

Julkunen, V., see Hartikainen, P. (4) 857-874

Juvés, S., see Aso, E. (2) 439-459

Kadish, I., see Searcy, J.L. (4) 943-961

Kalinine, E., see Muller, A.P. (4) 889-898

Kanakis, D., see Herring, A. (4) 963-979

Ke, W., see Borbon, I. (4) 875-887

Keller, J.N., see Bruce-Keller, A.J. (4) 899-908

Keyvani, K., see Herring, A. (4) 963-979

Kirsch, W.M., see Prasanthi, J.R.P. (1) 167-182

Kirsch, W.M., see Zabel, M. (2) 311-321

Kittus, R., see Rohrer, J.D. (2) 407-411 
Kivipelto, M., see Hartikainen, P. (4) 857-874

Kłoszewska, I., see Lunnon, K. (3) 685-710

Klugman, A., D.P. Naughton, M. Isaac, I. Shah, A. Petroczi and N. Tabet, Antioxidant Enzymatic Activities in Alzheimer's Disease: The Relationship to Acetylcholinesterase Inhibitors (3) 467-474

Koedam, E.L.G.E., see Smits, L.L. (1) 101-108

Koene, T., see Smits, L.L. (1) 101-108

Kölble, K., see Herring, A. (4) 963-979

Kretzschmar, H., see Schmidt, C. (4) 751-756

Kuller, L.H., see Hughes, T.M. (1) 53-61

Kuo, C.-C., see Zhang, C. (4) 919-934

Lan, Y., see Yu, Y. (2) 263-275

Landfield, P.W., see Searcy, J.L. (4) 943-961

Lannes, B., see Wallon, D. (4) 847-856

Lanni, C., see Buoso, E. (2) 393-405

Laplanche, J.-L., see Schmidt, C. (4) 751-756

Laquerrière, A., see Wallon, D. (4) 847-856

Lauritzen, I., see Flammang, B. (1) 145-153

Le Ber, I., see Wallon, D. (4) 847-856

Lee, H.-g., see Casadesús, G. (3) 573-583

Lewis, S.J.G., see Mowszowski, L. (1) 209-219

Li, K., see Liang, P. (3) 475-487

Li, R., see Long, J. (3) 545-558

Liang, P., Z. Wang, Y. Yang and K. Li, Three Subsystems of the Inferior Parietal Cortex Are Differently Affected in Mild Cognitive Impairment (3) 475487

Limon, D., see Diaz, A. (3) 505-522

Lin, C.-P., see Wang, P.-N. (2) 423-437

Lin, K.-N., see Wang, P.-N. (2) 423-437

Lin, X., see Cao, C. (3) 559-572

Lindberg, O., M. Walterfang, J.C.L. Looi, N. Malykhin, P. Östberg, B. Zandbelt, M. Styner, B. Paniagua, D. Velakoulis, E. Örndahl and L.-O. Wahlund, Hippocampal Shape Analysis in Alzheimer's Disease and Frontotemporal Lobar Degeneration Subtypes (2) 355-365

Lindsley, T.A., see Mathew, A. (3) 617-627

Lirng, J.-F., see Wang, P.-N. (2) 423-437

Liu, G.-P., see Chen, N.-N. (3) 585-594

Liu, X., see Yu, Y. (2) 263-275

Liu, Y., see Yu, Y. (2) 263-275

Lladó, A., see Balasa, M. (3) 605-616

Loewenstein, D.A., see Cao, C. (3) 559-572

Long, J., P. He, Y. Shen and R. Li, New Evidence of Mitochondria Dysfunction in the Female Alzheimer's Disease Brain: Deficiency of Estrogen Receptor- $\beta$ (3) 545-558

Looi, J.C.L., see Lindberg, O. (2) 355-365
Lopez, O.L., see Hughes, T.M. (1) 53-61

Lossi, L., see Alasia, S. (1) 41-51

Lourdusamy, A., see Lunnon, K. (3) 685-710

Lovestone, S., see Lunnon, K. (3) 685-710

Lu, K.P., see Pastorino, L. (2) 277-297

Luchsinger, J.A., see Geerlings, M.I. (1) 75-82

Lunnon, K., Z. Ibrahim, P. Proitsi, A. Lourdusamy, S. Newhouse, M. Sattlecker, S. Furney, M. Saleem, H. Soininen, I. Kłoszewska, P. Mecocci, M. Tsolaki, B. Vellas, G. Coppola, D. Geschwind, A. Simmons, S. Lovestone, R. Dobson and A. Hodges, Mitochondrial Dysfunction and Immune Activation are Detectable in Early Alzheimer's Disease Blood (3) 685-710

Luo, D.-J., see Chen, N.-N. (3) 585-594

Lupoli, S., see Albani, D. (4) 745-749

Ma, S.L., see Pastorino, L. (2) 277-297

Magnani, G., see Albani, D. (4) 745-749

Maldonado, R., see Aso, E. (2) 439-459

Malykhin, N., see Lindberg, O. (2) 355-365

Mandel, S.A., see Zheng, H. (1) 1-16

Mariani, C., see Albani, D. (4) 745-749

Mariani, S., see Albani, D. (4) 745-749

Martens, E.G.H.J., see Gommer, E.D. (4) 805-813

Martinaud, O., see Wallon, D. (4) 847-856

Martínez, E., A. Navarro, C. Ordóñez, E. del Valle and J. Tolivia, Amyloid- $\beta_{25-35}$ Induces Apolipoprotein D Synthesis and Growth Arrest in HT22 Hippocampal Cells (2) 233-244

Martinez-Martin, P., see Schmidt, C. (4) 751-756

Martín-Requero, Á., see Esteras, N. (2) 337-353

Marwarha, G., see Prasanthi, J.R.P. (1) 167-182

Masters, C.L., see Watt, A.D. (2) 323-336

Mathew, A., T.A. Lindsley, A. Sheridan, D.L. Bhoiwala, S.F. Hushmendy, E.J. Yager, E.A. Ruggiero and D.R. Crawford, Degraded Mitochondrial DNA is a Newly Identified Subtype of the Damage Associated Molecular Pattern (DAMP) Family and Possible Trigger of Neurodegeneration (3) 617-627

Mattace-Raso, F.U.S., see Verheij, S. (1) 131-143

Mattsson, N., E. Portelius, S. Rolstad, M. Gustavsson, U. Andreasson, M. Stridsberg, A. Wallin, K. Blennow and H. Zetterberg, Longitudinal Cerebrospinal Fluid Biomarkers over Four Years in Mild Cognitive Impairment (4) 767-778

Mauleón, A., see Alegret, M. (1) 109-120

Maurage, C.-A., see Rollin-Sillaire, A. (4) 833-845

Mayeux, R., see Geerlings, M.I. (1) 75-82

McNay, E.C., see Pearson-Leary, J. (2) 413-422 
Mecocci, P., see Lunnon, K. (3) 685-710

Melo-Pires, M., see Taipa, R. (1) 83-90

Ménard, M., see Chakravarthy, B. (3) 675-684

Merighi, A., see Alasia, S. (1) 41-51

Mess, W.H., see Gommer, E.D. (4) 805-813

Messis, C., see Sabbagh, M.N. (3) 711-727

Miao, J., see Wei, L. (3) 531-543

Milterberger-Miltenyi, G., see Taipa, R. (1) 83-90

Milward, E.A., see Johnstone, D.M. (4) 791-803

Molinuevo, J.-L., see Balasa, M. (3) 605-616

Moreaud, O., see Wallon, D. (4) 847-856

Mortimer, J.A., D. Ding, A.R. Borenstein, C. DeCarli, Q. Guo, Y. Wu, Q. Zhao and S. Chu, Changes in Brain Volume and Cognition in a Randomized Trial of Exercise and Social Interaction in a CommunityBased Sample of Non-Demented Chinese Elders (4) 757-766

Morvan, J., see Rollin-Sillaire, A. (4) 833-845

Moscato, P., see Johnstone, D.M. (4) 791-803

Moshier, E., see Ravona-Springer, R. (2) 299-309

Mouiha, A., S. Duchesne, Towards a Dynamic Biomarker Model in Alzheimer's Disease (1) 91-100

Mowszowski, L., D.F. Hermens, K. Diamond, L. Norrie, I.B. Hickie, S.J.G. Lewis and S.L. Naismith, Reduced Mismatch Negativity in Mild Cognitive Impairment: Associations with Neuropsychological Performance (1) 209-219

Mueller, C., see Zabel, M. (2) 311-321

Muilwijk, D., see Verheij, S. (1) 131-143

Muller, A.P., E.R. Zimmer, E. Kalinine, C.B. Haas, J.P. Oses, A.M. de Assis, A. Galina, D.O. Souza and L.V. Portela, Physical Exercise Exacerbates Memory Deficits Induced by Intracerebroventricular STZ but Improves Insulin Regulation of $\mathrm{H}_{2} \mathrm{O}_{2}$ Production in Mice Synaptosomes (4) 889-898

Muñoz, F.J., see Aso, E. (2) 439-459

Murphy, M.P., see Searcy, J.L. (4) 943-961

Murray, A.D., see Whalley, L.J. (2) 253-261

Murray, I.V.J., see Fawver, J.N. (1) 63-73

Nagai, Y., see Zhou, B. (2) 367-375

Naismith, S.L., see Mowszowski, L. (1) 209-219

Nakatani, E., see Zhou, B. (2) 367-375

Nandi, S., see Friedland, R.P. (3) 729-730

Nation, D.A., L. Delano-Wood, K.J. Bangen, C.E. Wierenga, A.J. Jak, L.A. Hansen, D.R. Galasko, D.P. Salmon and M.W. Bondi, Antemortem Pulse Pressure Elevation Predicts Cerebrovascular Disease in Autopsy-Confirmed Alzheimer's Disease (3) 595-603

Naughton, D.P., see Klugman, A. (3) 467-474
Navarro, A., see Martínez, E. (2) 233-244

Neumeister, K.L., M.W. Riepe, Synergistic Effects of Antidementia Drugs on Spatial Learning and Recall in the APP23 Transgenic Mouse Model of Alzheimer's Disease (2) 245-251

Newhouse, S., see Lunnon, K. (3) 685-710

Nicholson, L., see Pastorino, L. (2) 277-297

Nie, G., see Wan, L. (3) 523-530

Niskanen, E., see Hartikainen, P. (4) 857-874

Noel-Hudson, M.-S., see Do, T.M. (1) 155-166

Norrie, L., see Mowszowski, L. (1) 209-219

Nye, D.M., see Bruce-Keller, A.J. (4) 899-908

O’Bryant, S.E., see Royall, D.R. (3) 639-649

Olynyk, J.K., see Johnstone, D.M. (4) 791-803

Ordóñez, C., see Martínez, E. (2) 233-244

Örndahl, E., see Lindberg, O. (2) 355-365

Ortuño-Sahagún, D., see Casadesús, G. (3) 573-583

Oses, J.P., see Muller, A.P. (4) 889-898

Östberg, P., see Lindberg, O. (2) 355-365

Ou, F., see Wang, X. (3) 665-673

Ourselin, S., see Rohrer, J.D. (2) 407-411

Pahnke, J., see Schmidt, A. (3) 651-664

Pallàs, M., see Casadesús, G. (3) 573-583

Palmer, R.F., see Royall, D.R. (3) 639-649

Palomer, E., see Aso, E. (2) 439-459

Pancani, T., see Searcy, J.L. (4) 943-961

Pandya, D., see Pastorino, L. (2) 277-297

Panetta, V., see Ventriglia, M. (4) 981-984

Paniagua, B., see Lindberg, O. (2) 355-36

Paquet, C., see Wallon, D. (4) 847-856

Pardossi-Piquard, R., see Flammang, B. (1) 145-153

Pariente, J., see Wallon, D. (4) 847-856

Pasquier, F., see Rollin-Sillaire, A. (4) 833-845

Pasquier, F., see Wallon, D. (4) 847-856

Pastorino, L., S.L. Ma, M. Balastik, P. Huang, D. Pandya, L. Nicholson and K.P. Lu, Alzheimer's Disease-Related Loss of Pin1 Function Influences the Intracellular Localization and the Processing of A $\beta P P$ (2) 277-297

Paulus, W., see Herring, A. (4) 963-979

Pearson-Leary, J., E.C. McNay, Intrahippocampal Administration of Amyloid- $\beta_{1-42}$ Oligomers Acutely Impairs Spatial Working Memory, Insulin Signaling, and Hippocampal Metabolism (2) 413-422

Pel, J.J.M., see Verheij, S. (1) 131-143

Pereira, S., see Taipa, R. (1) 83-90

Perez, K.A., see Watt, A.D. (2) 323-336

Perry, G., Journal of Huntington's Disease - The Family Gets Bigger (4) 735 
Petersen, F., see Zabel, M. (2) 311-321

Petroczi, A., see Klugman, A. (3) 467-474

Phelps, J.T., see Searcy, J.L. (4) 943-961

Pijnenburg, Y.A.L., see Smits, L.L. (1) 101-108

Pinto, P.S., see Taipa, R. (1) 83-90

Popovic, J., see Searcy, J.L. (4) 943-961

Popovics, P., A.J. Stewart, Phospholipase C- $\eta$ Activity may Contribute to Alzheimer's Disease-Associated Calciumopathy (4) 737-744

Portela, L.V., see Muller, A.P. (4) 889-898

Portelius, E., see Mattsson, N. (4) 767-778

Porter, N.M., see Searcy, J.L. (4) 943-961

Potter, H., see Cao, C. (3) 559-572

Prá, I.D., see Chakravarthy, B. (3) 675-684

Prasanthi, J.R.P., M. Schrag, B. Dasari, G. Marwarha, A. Dickson, W.M. Kirsch and O. Ghribi, Deferiprone Reduces Amyloid- $\beta$ and Tau Phosphorylation Levels but not Reactive Oxygen Species Generation in Hippocampus of Rabbits Fed a Cholesterol-Enriched Diet (1) 167-182

Proitsi, P., see Lunnon, K. (3) 685-710

Puel, M., see Wallon, D. (4) 847-856

Quillard-Muraine, M., see Wallon, D. (4) 847-856

Rábano, A., see Schmidt, C. (4) 751-756

Racchi, M., see Buoso, E. (2) 393-405

Ramakers, I.H.G.B., see Gommer, E.D. (4) 805-813

Ramassamy, C., see Doggui, S. (2) 377-392

Rapp, M., see Ravona-Springer, R. (2) 299-309

Räsänen, J., see Hartikainen, P. (4) 857-874

Ravona-Springer, R., E. Moshier, J. Schmeidler, J. Godbold, J. Akrivos, M. Rapp, H.T. Grossman, M. Wysocki, J.M. Silverman, V. Haroutunian and M.S. Beeri, Changes in Glycemic Control are Associated with Changes in Cognition in NonDiabetic Elderly (2) 299-309

Recuero, M., see Santana, S. (4) 815-831

Rembach, A.R., see Watt, A.D. (2) 323-336

Remes, A.M., see Hartikainen, P. (4) 857-874

Remington, R., see Shea, T.B. (1) 27-33

Reulen, J.P.H., see Gommer, E.D. (4) 805-813

Reuling, I.E.W., see Smits, L.L. (1) 101-108

Ribes, S., see Do, T.M. (1) 155-166

Riepe, M.W., see Neumeister, K.L. (2) 245-251

Ritchie, K., see Ancelin, M.-L. (3) 629-637

Riveros, C., see Johnstone, D.M. (4) 791-803

Roca, I., see Alegret, M. (1) 109-120

Roeber, S., see Schmidt, C. (4) 751-756

Rogers, E., see Shea, T.B. (1) 27-33

Rohrer, J.D., M.J. Clarkson, R. Kittus, M.N. Rossor, S. Ourselin, J.D. Warren and N.C. Fox, Rates of
Hemispheric and Lobar Atrophy in the Language Variants of Frontotemporal Lobar Degeneration (2) 407-411

Rollin-Sillaire, A., S. Bombois, V. Deramecourt, A. Steinert-Emptaz, J. Salleron, J. Morvan, C.-A. Maurage, M. Steinling and F. Pasquier, Contribution of Single Photon Emission Computed Tomography to the Differential Diagnosis of Dementia in a Memory Clinic (4) 833-845

Rollin-Sillaire, A., see Wallon, D. (4) 847-856

Rolstad, S., see Mattsson, N. (4) 767-778

Rosano, C., see Hughes, T.M. (1) 53-61

Rosende-Roca, M., see Alegret, M. (1) 109-120

Rossor, M.N., see Rohrer, J.D. (2) 407-411

Rouaud, O., see Ancelin, M.-L. (3) 629-637

Rousseau, S., see Wallon, D. (4) 847-856

Rovelet-Lecrux, A., see Wallon, D. (4) 847-856

Royall, D.R., R.F. Palmer and S.E. O'Bryant, Validation of a Latent Variable Representing the Dementing Process (3) 639-649

Ruggiero, E.A., see Mathew, A. (3) 617-627

Ruíz, A., see Alegret, M. (1) 109-120

Sabbagh, M.N., C. Messis, R.P. Friedland and C. Geula, The 5th International Conference on Alzheimer's Disease and Related Disorders in the Middle East, 15-17 May 2009, Limassol Cyprus (3) 711-727

Sachdev, P., see Bunce, D. (4) 935-942

Sahni, J.K., see Doggui, S. (2) 377-392

Saint-Pol, J., E. Vandenhaute, M.-C. Boucau, P. Candela, L. Dehouck, R. Cecchelli, M.-P. Dehouck, L. Fenart and F. Gosselet, Brain Pericytes ABCA1 Expression Mediates Cholesterol Efflux but not Cellular Amyloid- $\beta$ Peptide Accumulation (3) 489-503

Sakurai, T., see Wang, X. (3) 665-673

Saleem, M., see Lunnon, K. (3) 685-710

Salleron, J., see Rollin-Sillaire, A. (4) 833-845

Salmon, D.P., see Nation, D.A. (3) 595-603

Sánchez-Valle, R., see Balasa, M. (3) 605-616

Santana, S., M.J. Bullido, M. Recuero, F. Valdivieso and J. Aldudo, Herpes Simplex Virus Type I Induces an Incomplete Autophagic Response in Human Neuroblastoma Cells (4) 815-831

Sarazin, M., see Wallon, D. (4) 847-856

Satoh, K., see Schmidt, C. (4) 751-756

Sattlecker, M., see Lunnon, K. (3) 685-710

Sauvée, M., see Wallon, D. (4) 847-856

Scarpini, E., see Albani, D. (4) 745-749

Schall, H.E., see Fawver, J.N. (1) 63-73

Scheltens, P., see Smits, L.L. (1) 101-108 
Scherrmann, J.-M., see Do, T.M. (1) 155-166

Schettini, G., see Buoso, E. (2) 393-405

Schmeidler, J., see Ravona-Springer, R. (2) 299-309

Schmidt, A., J. Pahnke, Efficient Near-Infrared In Vivo Imaging of Amyoid- $\beta$ Deposits in Alzheimer's Disease Mouse Models (3) 651-664

Schmidt, C., S. Haïk, K. Satoh, A. Rábano, P. MartinezMartin, S. Roeber, J.-P. Brandel, M. Calero-Lara, J. de Pedro-Cuesta, J.-L. Laplanche, J.-J. Hauw, H. Kretzschmar and I. Zerr, Rapidly Progressive Alzheimer's Disease: A Multicenter Update (4) 751-756

Schofield, E., see Cao, C. (3) 559-572

Schrag, M., see Prasanthi, J.R.P. (1) 167-182

Schrag, M., see Zabel, M. (2) 311-321

Schupf, N., see Geerlings, M.I. (1) 75-82

Scott, R.J., see Johnstone, D.M. (4) 791-803

Searcy, J.L., J.T. Phelps, T. Pancani, I. Kadish, J. Popovic, K.L. Anderson, T.L. Beckett, M.P. Murphy, K.-C. Chen, E.M. Blalock, P.W. Landfield, N.M. Porter and O. Thibault, LongTerm Pioglitazone Treatment Improves Learning and Attenuates Pathological Markers in a Mouse Model of Alzheimer's Disease (4) 943-961

Sellal, F., see Wallon, D. (4) 847-856

Serot, J.-M., J. Zmudka and P. Jouanny, A Possible Role for CSF Turnover and Choroid Plexus in the Pathogenesis of Late Onset Alzheimer's Disease (1) $17-26$

Sevalle, J., see Flammang, B. (1) 145-153

Shah, I., see Klugman, A. (3) 467-474

Sharma, S., see Whalley, L.J. (2) 253-261

Sharpe, L.J., J. Wong, B. Garner, G.M. Halliday and A.J. Brown, Is Seladin-1 Really a Selective Alzheimer's Disease Indicator? (1) 35-39

Shea, T.B., E. Rogers and R. Remington, Nutrition and Dementia: Are we Asking the Right Questions? (1) 27-33

Shen, Y., see Long, J. (3) 545-558

Sheridan, A., see Mathew, A. (3) 617-627

Shih, D.M., see Borowczyk, K. (2) 225-231

Shijaku, E., see Gommer, E.D. (4) 805-813

Silverman, J.M., see Ravona-Springer, R. (2) 299-309

Simmons, A., see Lunnon, K. (3) 685-710

Small, B., see Cao, C. (3) 559-572

Small, S.A., see Geerlings, M.I. (1) 75-82

Smirnova, M., see Do, T.M. (1) 155-166

Smith, M.A., see Casadesús, G. (3) 573-583

Smits, L.L., Y.A.L. Pijnenburg, E.L.G.E. Koedam, A.E. van der Vlies, I.E.W. Reuling, T. Koene, C.E. Teunissen, P. Scheltens and W.M. van der Flier,
Early Onset Alzheimer's Disease is Associated with a Distinct Neuropsychological Profile (1) 101-108

Soininen, H., see Hartikainen, P. (4) 857-874

Soininen, H., see Lunnon, K. (3) 685-710

Souza, D.O., see Muller, A.P. (4) 889-898

Squitti, R., see Albani, D. (4) 745-749

Squitti, R., see Ventriglia, M. (4) 981-984

Staff, R.T., see Whalley, L.J. (2) 253-261

Starr, J.M., see Whalley, L.J. (2) 253-261

Steiner, K.M., see Herring, A. (4) 963-979

Steinert-Emptaz, A., see Rollin-Sillaire, A. (4) 833-845

Steinling, M., see Rollin-Sillaire, A. (4) 833-845

Stewart, A.J., see Popovics, P. (4) 737-744

Streichenberger, N., see Wallon, D. (4) 847-856

Stridsberg, M., see Mattsson, N. (4) 767-778

Styner, M., see Lindberg, O. (2) 355-365

Sun, M., see Yu, Y. (2) 263-275

Sutton-Tyrrell, K., see Hughes, T.M. (1) 53-61

Tabet, N., see Klugman, A. (3) 467-474

Taipa, R., A. Tuna, J. Damásio, P.S. Pinto, S. Cavaco, S. Pereira, G. Milterberger-Miltenyi, D. Galimberti and M. Melo-Pires, Clinical, Neuropathological, and Genetic Characteristics of the Novel IVS9+1delG GRN Mutation in a Patient with Frontotemporal Dementia (1) 83-90

Tajes, M., see Casadesús, G. (3) 573-583

Takata, T., see Wang, X. (3) 665-673

Tárraga, L., see Alegret, M. (1) 109-120

Teramukai, S., see Zhou, B. (2) 367-375

Teunissen, C.E., see Smits, L.L. (1) 101-108

Thévenet, A., see Flammang, B. (1) 145-153

Thibault, O., see Searcy, J.L. (4) 943-961

Thomas-Antérion, C., see Wallon, D. (4) 847-856

Tolivia, J., see Martínez, E. (2) 233-244

Totenhagen, J., see Borbon, I. (4) 875-887

Trinder, D., see Johnstone, D.M. (4) 791-803

Trouard, T., see Borbon, I. (4) 875-887

Tsolaki, M., see Lunnon, K. (3) 685-710

Tu, P., see Yu, Y. (2) 263-275

Tudor-Locke, C., see Bruce-Keller, A.J. (4) 899-908

Tuna, A., see Taipa, R. (1) 83-90

Uylings, H.B.M., see Echávarri, C. (4) 909-917

Valdivieso, F., see Guzman-Sanchez, F. (4) 779-790

Valdivieso, F., see Santana, S. (4) 815-831

Valero, S., see Alegret, M. (1) 109-120

van der Cammen, T.J.M., see Verheij, S. (1) 131-143

van der Flier, W.M., see Smits, L.L. (1) 101-108 
van der Steen, J., see Verheij, S. (1) 131-143

van der Vlies, A.E., see Smits, L.L. (1) 101-108

Vandenhaute, E., see Saint-Pol, J. (3) 489-503

Vanninen, R., see Hartikainen, P. (4) 857-874

Velakoulis, D., see Lindberg, O. (2) 355-365

Vellas, B., see Lunnon, K. (3) 685-710

Ventriglia, M., S. Bucossi, V. Panetta and R. Squitti, Copper in Alzheimer's Disease: A Meta-Analysis of Serum, Plasma, and Cerebrospinal Fluid Studies (4) 981-984

Verheij, S., D. Muilwijk, J.J.M. Pel, T.J.M. van der Cammen, F.U.S. Mattace-Raso and J. van der Steen, Visuomotor Impairment in Early-Stage Alzheimer's Disease: Changes in Relative Timing of Eye and Hand Movements (1) 131-143

Verhey, F.R.J., see Echávarri, C. (4) 909-917

Verhey, F.R.J., see Gommer, E.D. (4) 805-813

Vidal-Piñeiro, D., see Balasa, M. (3) 605-616

Villemagne, V.L., see Watt, A.D. (2) 323-336

Vinyes-Junqué, G., see Alegret, M. (1) 109-120

Wahlund, L.-O., see Lindberg, O. (2) 355-365

Wallin, A., see Mattsson, N. (4) 767-778

Wallon, D., S. Rousseau, A. Rovelet-Lecrux, M. Quillard-Muraine, L. Guyant-Maréchal, O. Martinaud, J. Pariente, M. Puel, A. RollinSillaire, F. Pasquier, I. Le Ber, M. Sarazin, B. Croisile, C. Boutoleau-Bretonnière, C. ThomasAntérion, C. Paquet, O. Moreaud, A. Gabelle, F. Sellal, M. Sauvée, A. Laquerrière, C. Duyckaerts, M.-B. Delisle, N. Streichenberger, B. Lannes, T. Frebourg, D. Hannequin and D. Campion, The French Series of Autosomal Dominant Early Onset Alzheimer's Disease Cases: Mutation Spectrum and Cerebrospinal Fluid Biomarkers (4) 847-856

Walterfang, M., see Lindberg, O. (2) 355-365

Wan, L., G. Nie, J. Zhang and B. Zhao, Overexpression of Human Wild-Type Amyloid- $\beta$ Protein Precursor Decreases the Iron Content and Increases the Oxidative Stress of Neuroblastoma SH-SY5Y Cells (3) 523-530

Wang, H., see Yu, Y. (2) 263-275

Wang, J.-Z., see Chen, N.-N. (3) 585-594

Wang, L., see Cao, C. (3) 559-572

Wang, P., see Wei, L. (3) 531-543

Wang, P.-N., K.-H. Chou, J.-F. Lirng, K.-N. Lin, W.-T. Chen and C.-P. Lin, Multiple Diffusivities Define White Matter Degeneration in Amnestic Mild Cognitive Impairment and Alzheimer's Disease (2) 423-437

Wang, Q., see Chen, N.-N. (3) 585-594
Wang, X., T. Takata, X. Bai, F. Ou, K. Yokono and T. Sakurai, Pyruvate Prevents the Inhibition of the Long-term Potentiation Induced by Amyloid- $\beta$ through Protein Phosphatase 2A Inactivation (3) 665-673

Wang, Y., see Chen, N.-N. (3) 585-594

Wang, Z., see Liang, P. (3) 475-487

Warren, J.D., see Rohrer, J.D., (2) 407-411

Watt, A.D., K.A. Perez, A.R. Rembach, C.L. Masters, V.L. Villemagne and K.J. Barnham, Variability in Blood-Based Amyloid- $\beta$ Assays: The Need for Consensus on Pre-Analytical Processing (2) 323336

Wei, L., H. Yang, Z. Xie, S. Yang, H. Yang, C. Zhao, P. Wang, S. Xu, J. Miao, B. Zhao and J. Bi, A Butyrolactone Derivative 3BDO Alleviates Memory Deficits and Reduces Amyloid- $\beta$ Deposition in an A $\beta$ PP/PS1 Transgenic Mouse Model (3) 531-543

Whalley, L.J., S. Sharma, H.C. Fox, A.D. Murray, R.T. Staff, A.C. Duthie, I.J. Deary and J.M. Starr, Anticholinergic Drugs in Late Life: Adverse Effects on Cognition but not on Progress to Dementia (2) 253-261

Whitehouse, P., Book Review (1) 221

Whitfield, J., see Chakravarthy, B. (3) 675-684

Widera, M.P., see Herring, A. (4) 963-979

Wierenga, C.E., see Nation, D.A. (3) 595-603

Wong, J., see Sharpe, L.J. (1) 35-39

Wu, Y., see Cao, C. (3) 559-572

Wu, Y., see Mortimer, J.A. (4) 757-766

Wysocki, M., see Ravona-Springer, R. (2) 299-309

Xiao, R., see Yu, Y. (2) 263-275

Xie, Z., see Wei, L. (3) 531-543

Xu, S., see Wei, L. (3) 531-543

Xu, X., see Clausen, A. (1) 183-208

Yager, E.J., see Mathew, A. (3) 617-627

Yang, H., see Wei, L. (3) 531-543

Yang, H., see Wei, L. (3) 531-543

Yang, S., see Wei, L. (3) 531-543

Yang, Y., see Liang, P. (3) 475-487

Yao, X.-Q., see Chen, N.-N. (3) 585-594

Yokono, K., see Wang, X. (3) 665-673

Youdim, M.B.H., see Zheng, H. (1) 1-16

Yu, C., see Chen, N.-N. (3) 585-594

Yu, Y., L. Zhou, M. Sun, T. Zhou, K. Zhong, H. Wang, Y. Liu, X. Liu, R. Xiao, J. Ge, P. Tu, D.S. Fan, Y. Lan, C. Hui and D. Chui, Xylocoside G Reduces Amyloid- $\beta$ Induced Neurotoxicity by Inhibiting 
NF- $\kappa B$ Signaling Pathway in Neuronal Cells (2) 263-275

Zabel, M., M. Schrag, C. Mueller, W. Zhou, A. Crofton, F. Petersen, A. Dickson and W.M. Kirsch, Assessing Candidate Serum Biomarkers for Alzheimer's Disease: A Longitudinal Study (2) 311-321

Zandbelt, B., see Lindberg, O. (2) 355-365

Zenteno, E., see Diaz, A. (3) 505-522

Zerr, I., see Schmidt, C. (4) 751-756

Zetterberg, H., see Mattsson, N. (4) 767-778

Zhang, C., C.-C. Kuo, A.W.L. Chiu and J. Feng, Prediction of $S$-glutathionylated Proteins Progression in Alzheimer's Transgenic Mouse Model using Principle Component Analysis (4) 919-934

Zhang, C., see Cao, C. (3) 559-572

Zhang, J., see Wan, L. (3) 523-530

Zhao, B., see Wan, L. (3) 523-530

Zhao, B., see Wei, L. (3) 531-543
Zhao, C., see Wei, L. (3) 531-543

Zhao, Q., see Mortimer, J.A. (4) 757-766

Zheng, H., T. Amit, O. Bar-Am, M. Fridkin, M.B.H. Youdim and S.A. Mandel, From AntiParkinson's Drug Rasagiline to Novel Multitarget Iron Chelators with Acetylcholinesterase and Monoamine Oxidase Inhibitory and Neuroprotective Properties for Alzheimer's Disease (1) 1-16

Zhong, K., see Yu, Y. (2) 263-275

Zhou, B., E. Nakatani, S. Teramukai, Y. Nagai and M. Fukushim, Risk Classification in Mild Cognitive Impairment Patients for Developing Alzheimer's Disease (2) 367-375

Zhou, L., see Yu, Y. (2) 263-275

Zhou, T., see Yu, Y. (2) 263-275

Zhou, W., see Zabel, M. (2) 311-321

Zhu, X., see Fawver, J.N. (1) 63-73

Zimmer, E.R., see Muller, A.P. (4) 889-898

Zmudka, J., see Serot, J.-M. (1) 17-26 